\title{
Storing and accessing the largest astronomical catalogues with the SAI CAS project
}

\author{
Sergey E. Koposov ${ }^{1,2,3}$, Oleg Bartunov ${ }^{2}$ and Sergey Karpov ${ }^{4}$ \\ ${ }^{1}$ Max Planck Institute for Astronomy, Königstuhl 17, Heidelberg, D-69117, Germany \\ ${ }^{2}$ Sternberg Astronomical Institute, Universitetskiy pr. 13, Moscow, 119992, Russia \\ ${ }^{3}$ Institute of Astronomy, Madingley Road, Cambridge CB3 0HA, UK \\ ${ }^{4}$ Special Astrophysical Observatory, pos. Nijniy Arkhyz, Russia \\ email: math@sai.msu.ru
}

One of the main goals of the Virtual Observatory activities right now is to provide the simple and powerful access to the large existing astronomical datasets in the VO compatible way. That is why we want present the results of the recent development of Sternberg Astronomical Institute Catalogue Access Services (SAI CAS) project - the first and the only project in Russia, which provides on-line access to the major astronomical catalogues and different services on top of them. It is developed by a group of astronomers in a framework of SAI Astronet project, funded by Russian Foundation for Basic Research. SAI CAS is an open-source implementation of the general Catalogue access service (influenced by SDSS CASjobs \& OpenSkyQuery projects), based on original algorithms and open-source software. We decided to build our own system providing an effective access to the major astronomical catalogues and different services including cone-searches and cross-matching of user data with hosted catalogues.

We use only open-source software with good support and stable history of development - Apache Tomcat, Axis, PostgreSQL and Java. SAI CAS consists of the database server running PostgreSQL 8.1+, which stores the catalogues and all their metadata, the application server with set of web-services and a pool of JDBC connections to database server, and the frontend for the interaction with users and web-interface.

Clients could be web browsers or any programs communicating with SAI CAS via HTTP and SOAP protocols and retrieving the data in different formats (VOTable, CSV, etc.). The spatial queries (cross-matches and cone-searches) in the database are operated using the developed by us Q3C sky indexing scheme and corresponding package for PostgreSQL v.8.1+ which is freely available. It provides the very fast access to the data with spherical attributes and combines advantages of well-known HTM and HEALPIX indexing schemes. Currently we provide the ConeSearch service (registered in the VO registry) for USNO-A2/B1, 2MASS, UCAC-2, NOMAD, DENIS, Tycho-2, 2XMM (SDSS DR5 will be loaded very soon). These catalogues are also available for the crossmatches. It is possible to perform the crossmatches of the supplied by user VOTable with one of the hosted catalogues.

All the SAI CAS system can be easily operated with the set of web services, with which the data can be uploaded to the system, the data and the metadata can be queried and updated. The formal Skynode interface of querying our catalogues is being built. The SAI CAS system is mainly targeted for science problem solving, and currently it is already used by several science projects in Russia. The web-site of the SAI CAS project is:

<http://vo.astronet.ru>. 